\title{
Self-care Management and Education of Diabetic Clients
}

\author{
Article by Marrie L Davis \\ Ph.D, Texila American University, Saint Vincent and the Grenadines \\ E-mail: marriedavis63@hotmail.com
}

\begin{abstract}
The roles and responsibilities of clients in managing non-communicable diseases (NCDs) remain vital. Diabetes mellitus is a chronic disease that affects people from underdeveloped, developing and developed countries despite stages of economic and social development. Diabetes self-management education (DSME) is an ongoing process of facilitating the knowledge, other skills necessary for self-care and incorporating the critical processes such as the needs affected goals and care guided by evidence-based standards.

The overall goals of the article supported informed decision-making, self-care behaviors, problem-solving and collaborated with health care teams to improve clinical outcomes, enhanced health status and the quality of life living with diabetes. The self-care management model was designed to help clients living with the disease. The model provided supports and educated client on how to best adjust their lifestyle practices to sustain healthy living. The nursing process used as an assessment tool for designing the model. A client-friendly action plan and a simple take home card used to chart the way forward in managing self-care.

Technology has influenced many lives, which can be motivational and allowed for more frequent contact between healthcare providers and clients and enhanced self-care management behaviors and treatment plans. The application of scientific knowledge can be broadened to clients, homes, and communities, and provided individualized care and just-intime information.
\end{abstract}

Keywords: diabetes, self-care management, diabetes self-management education, compliance, lifestyle modifications

\section{Introduction}

Diabetes mellitus (DM) is a chronic disease that affects people from underdeveloped, developing and developed countries despite stages of economic and social development. In spite of scientific advances and easy access to health care, the prevalence of diabetes continues to increase (Seuring, 2015). The roles and responsibilities of clients in managing non-communicable diseases (NCDs) are vital. Therefore, self-care management and education improved health outcomes; however, knowledge must be evidence-based and relevant to the client's condition. Most important, knowledge must allow the client to assume a fundamental part in disease control and treatment. By improving clients' knowledge about the dangers, advantages and medical procedures in support to informed decision-making is paramount.

Self-care described as an evolutionary process of development or awareness living with the multifaceted nature of the disease (Paterson, 2000). Education is known as the wealth of knowledge acquired by an individual on a particular subject that provides an understanding of something or deposits of knowledge. Health education is consciously constructed opportunity for learning which involved communication designed to improve health literacy knowledge and developed life skills which are helpful to people and community health (WHO, 1998). Self-care management of diabetes involved day-to-day care and helps the client to live and function with the disease. Education enhances the understanding of the condition, selfadvocacy in deciding to act independently and to comply to care.

Diabetes self-management education (DSME) is an ongoing process of facilitating the knowledge, other skills necessary for self-care and incorporating the critical processes such as the needs affected goals and care guided by evidence-based standards. The overall goals of 
Texila International Journal of Nursing

Volume 3, Issue 1, Mar 2017

the article are to support informed decision-making, self-care behaviors, problem-solving and active collaboration with health care team to improve clinical outcomes, enhanced health status and the quality of life living with diabetes. Therefore, the designed model of self-care management will ably help clients living with the disease to sustain healthy day-to-day activities through lifestyle modifications, working with the healthcare team and compliance to medical therapy.

Key definitions: diabetes is a chronic metabolic non-communicable disease. The disease is evident once the pancreas is unable to produce the adequate amount of insulin or it is not capable of using insulin, resulting in the elevation of the blood glucose level (WHO, 1999)

Education is known as the wealth of knowledge acquired by an individual on a particular subject that provides the understanding of something or the deposits of knowledge.

Self-care management is the decisions and behaviors of clients with chronic illnesses and their engagement in what affect their health.

Diabetes self-management education (DSME) is an ongoing process of facilitating knowledge, other skills necessary for self-care and incorporating the necessary processes such as the needs affected goals and care guided by evidence-based standards.

Compliance describes the extent to which clients correctly follows medical advice such as medication or drug. It also applies to other situations such as medical devices used, self-care, self-directed exercises or therapy sessions (WHO, 2003).

Lifestyle modification entails changing of long-term habits, typically of eating or physical activity, adopting, maintaining and modifying behaviors for months, years, or for a lifetime.

\section{General goal of the article}

Optimal self-management entails the ability to monitor the disease and to build and use cognitive, behavioral, emotional strategies and a holistic approach improving the quality of life living with diabetes.

\section{Objectives of the article}

- Educate clients on the importance of complying and self-care management.

- Educate clients on the advantages of self-care management.

- Design a model of self-care management.

- Devise a take home passport or manual on self-care.

\section{Literature}

Diabetes mellitus (DM) is one of the oldest diseases known. About 3000 years ago the disease was first reported in Egyptian manuscript. Diabetes is one of the fastest developing chronic diseases known and has affected over 300 million people worldwide Matricciant, 2015). During 1936 the distinction was made between type one and type two DM (DM, 2011). The disease is characterized by hyperglycemia, insulin resistance and insulin deficiency (Maitra, 2005). Type $2 \mathrm{DM}$, is a chronic metabolic disorder and appears as an epidemic in some countries of the world. No cure was found for DM; however, treatment modalities included lifestyle modifications, treatment of obesity, oral hypoglycemic agents and insulin sensitizers.

The American Association of Diabetes Educators stressed the Seven Self-Care Behaviors framework for people living with diabetes should be skilled in and self-care behaviors to improve the quality of life and reduced associated complications (Boren, 2007). This author also affirmed the skills needed to carry out included blood glucose levels and blood pressure monitoring, eliminates smoking, foot self-checks, eyes and dental checks. The American Association of Diabetes Educators further forecasted seven essential self-care behaviors. These are healthy eating habits, being physically active, monitoring of blood glucose levels, complying with medications, rational problem-solving skills, healthy coping practices and less risky behaviors (AADE, 2008). The capacity to lower the gap between patient needs and access to health care service are aspects of self-care management (Barlow, 2002). 
On the other hand, one author felt that the quality of life could improve with people living with diabetes. The author affirmed self-care management and education helped in managing the condition (Grady, 2011). Self-care in diabetes has been described as a gradual process of development, knowledge or awareness of learning to survive with the complexities of diabetes (Cooper, 2003). However, the day-to-day care in diabetes is managed by the clients and their families. Therefore, it is a vital need for constant and valid measures for self-care management of the disease (Mc Nabb, 1997). There are many proposed measures suggested and useful for clinicians and educators managing diabetic people. It was reported that selfreport is one of the most practical and cost-effective approaches to self-care management. The seven behaviors identified, have found to be positively correlated with good glycemic control, reduction of complications and improvement in the quality of life (ADA, 2009).

Diabetes self-care requires the patients to make many dietary and lifestyle modifications supplemented with supportive role of healthcare team for maintaining a higher level of selfconfidence that leads to successful behavior change (Shobhana, 1999). Diabetic patients are expected to be knowledgeable and to follow a complex set of behavioral actions on a daily basis. Behavioral actions such as healthy lifestyle behaviors, after the recommended meal plan, engaging in physical activity, compliance to medication, monitoring blood glucose levels and responding to self-treating diabetes-related symptoms; foot-care guidance and seek medical care (Goodall, 1991).

The American Association of Clinical Endocrinologists accentuates the importance of patients becoming active and knowledgeable in their care (AACE, 2002). On the other hand, WHO has recognized the importance of patients learning to manage their diabetes (Hendra, 1997). The American Diabetes Association revised the standards of diabetes self-management education and findings revealed a four-fold increase in diabetic complications for people who had not received formal education about self-care practices (Mensing, 2006).

Self-management refers to the ability of clients, including their families, communities, healthcare professionals, to manage symptoms, treatment modalities, lifestyle changes, and psychosocial, cultural and spiritual consequences of health conditions. Also, self-management is described as the healthy lifestyle behaviors undertaken by people for optimal growth and development, or preventive strategies performed to promote or to support health (Richard, 2011). Therefore, ideal self-management entails the ability to monitor the disease and to develop and use cognitive, behavioral and emotional strategies to sustain a satisfactory quality of life.

Clients' knowledge improved health outcomes. The knowledge must be evidence-based and relevant to the patients. Most important, the knowledge must enable patients to assume an essential part in disease control and treatment. Hence, by improving patients' knowledge about risks, benefits and characteristics of medical procedures remain critical to support informed decision-making.

Studies on self-management education for adults with type-2 diabetes revealed improvement in glycemic control and observed benefits declined one to three months after the intervention ceased; these factors suggested that continuing education is necessary (Williams, 1998). Most importantly, reviews showed that diabetes self-management education revealed successes in lowering glycosylated hemoglobin levels (Norris, 2002). On the other hand, high-quality structured education has a philosophical effect on health outcomes and can significantly improve the quality of life. A Cochrane review affirmed that culturally, appropriate health education has short-to-medium term effects on glycemic control, the knowledge of diabetes and healthy lifestyles (Attridge, 2014).

\section{Model of care}

\section{Aims and assessment}

- Educate clients on the importance of monitoring and managing their diabetes.

- The ability to ably identify and to assess any problems in glycemic control and address them effectively. 
Texila International Journal of Nursing

Volume 3, Issue 1, Mar 2017

- Skillfully identify complications of diabetes and to obtain urgent treatment.

- Educate clients on the importance of healthy lifestyle practices.

- Assess clients' general health status and to provide treatment of associated or coincidental illness, physical, mental or others.

- Provide support and to educate clients on how to live with the disease and to best adjust their lifestyle practices to sustain healthy living.

\section{Complete assessment of client}

Assessment can be done at their homes, clinics, health facility or hospitals.

- Assessment includes physiological data, psychological, sociocultural, spiritual, and economic and lifestyle factors.

\section{Subjective data}

- History taking: demographic profile, the past, present and medical history, family history and other data needed.

- Obtain information from clients, relatives, next of kin, medical records, clinics, hospitals, medical practitioners and other sources to manage care.

\section{Objective data}

Use findings of physical examination to manage client's conditions.

- Record general observation during the assessment process and use findings to help in managing client.

- Diagnostic data obtained are analyzed

\section{Data analysis}

- Helps to find the real health problems

- Potential and high-risk health problems

- Also, helps to set goals centered on promoting and enhancing patients' level of wellness.

\section{Nursing diagnosis}

- The nursing diagnosis is made based on the real and potential problems identified. The plan of care is client-centered and holistic. Needs are prioritized based on the present needs affected, potential and high-risk health problems identified.

- Education centers on promoting, enhancing and modifying of healthy lifestyle activities, practices, and compliance with care.

\section{Plan of care}

- The goals must be short-term and long-term to manage the actual and potential problems.

- The goals must be specific, measurable, achievable, and realistic and time restricted.

- Each goal must be singular and relevant to the client's outcomes.

- The goals must be relevant and focus on a set of activities designed to improve client's actual problems and to prevent potential ones.

- These goals should provide the medical practitioners with a plan in which they can measure and evaluate client's improvement and collaboration of care.

- Intervention strategies are developed and communicated to the client, health care team and family to meet the unique circumstances of the client.

- The evidence-based care guide helps to addresses medications, diet, physical activities, self-monitoring and follow-up care.

- Modification of plans should be ongoing to discuss the affected needs.

\section{Client friendly action plan: Do it, share it and document it}

- Client oriented goals are activated.

- Goals are based on physiological and psychological needs affected. 
- The approach must be holistic.

- Independent interventions are implemented.

- Collaborative interventions are performed in conjunction with the family and other members of the healthcare team.

- Interventions are based on the priority of care.

- Direct and indirect care should be rendered.

- Ongoing data collection is essential to enhance care.

- Documentation of care is vital and in charting the way forward in managing care.

\section{Do it: Direct care}

- Care can be physical or verbal.

- Assist client with general activities of daily living.

- Education sessions are necessary and should be ongoing.

- Give feedback to client, medical team, and relatives.

\section{Indirect care}

- Ongoing monitoring of the client's general condition and improvement.

- Advocate on the client's behalf.

Nursing staff also is accountable; it is an important aspect and the legal requirement of nursing practice. Critical thinking skills helped to make the safest and most helpful choices for clients. Intellectual, interpersonal and technical skills are required in the decision-making process, safe and competent performance is required.

\section{Questions healthcare personnel asked during the caring process}

- Does the plan protect the safety of the client?

- Is the plan based on sound nursing knowledge and developed according to scientific problem-solving approach?

- Is the plan workable with the desired results?

- Is the plan prioritized based on the client's needs?

- Is the client actively involved, along with health team and relatives in the plan of care?

Share it: Interdisciplinary planning helps and offers an excellent method to coordinates care and interacts with other members of the healthcare team. This process helps to ensure effective planning, coordinating with other health care providers and assess care.

- The process focuses on ongoing data collection.

- Effective communication with the healthcare team, clients, and their relatives.

Document it: Documentation is a communication tool used. An effective means to exchange client's information. Documentation promotes effective communication between clients, caregivers, healthcare team, and their relatives. Documentation must be

- Accurate, clear, concise, comprehensive and confidential.

- Chronological and timely.

- Facilitate evidence-informed practice and a source for improving clients' outcomes. "Work not documented means it was not done" an old nursing quote.

- Quality nursing documentation promotes effective communication among caregivers which enhances quality care.

Evaluation: Measuring the effectiveness of assessing, analyzing, diagnosing, planning and implementing is done through the process of evaluation. The following steps are necessary to evaluate and reevaluate care. These steps can be done at any stage during the assessment process to ascertain progress.

- Analyzing clients' responses

- Identifying factors that contribute to success or failure.

- Determine the type of progress made in achieving the desired outcomes.

- Determine if the process is workable if not reassessment can be done to decide whether there is a need to change or to eliminate the goals or present action plan. 
Texila International Journal of Nursing

Volume 3, Issue 1, Mar 2017

- Regular evaluation is necessary it aids in determining the proper course of action to be taken identifies potential errors and ensures a smooth working process.

- This process aids in planning for ongoing and follow-up care.

Self-management, education, and compliance: Diabetic self-management education is the process of educating DM clients on how to manage their diabetes a vital aspect of clinical management. The self-management education model aims to increase knowledge, skills, and confidence leading to an informed decision regarding the disease. The goals of diabetic education are to optimize metabolic control, prevent acute and chronic complications, compliance and to enhance the quality of life. Also, to enable DM clients to increase control of their conditions and incorporate effective self-management into their daily activities of lives.

Effective self-management strategies require clients to have a sense of ownership of managing the disease. Self-management can be fostered through timely provision of information (Frost, 2014). On the other hand, high-quality structured education has a profound effect on health outcomes and can significantly improve the quality of life for people living with the disease.

\section{What all diabetic clients should be educated on?}

\section{The types of diabetes}

- Type 1 / Insulin-dependent diabetes mellitus (IDDM) or juvenile DM

- Type 2 or adult onset

- Gestational- onset during pregnancy

The path physiology of the disease: The cause of DM is multi-factorial and included both genetic and environmental elements that affect the beta-cell functions and tissues. DM also involved the muscles, liver, adipose tissue and the pancreas to insulin sensitivity. Diabetes occurs due to dis-balance between the demand and production of the hormone insulin. After eating or drinking the body breaks down sugars in the blood and converts it into glucose. The glucose travels through the bloodstream and provides the body with energy. The process is accomplished through the pancreas which produces the hormone insulin. The pancreas is an organ in the abdomen between the stomach and the spine and the remaining part in the small intestine. The pancreas produces the hormone insulin and secretes it into the bloodstream to regulate the body's glucose or sugar level.

People with diabetes the pancreas is either unable to use insulin effectively or produces too little insulin or none at all. The cells are deprived of their needed energy when the blood glucose level rises. Many problems are likely to occur affecting almost every part of the body. The common symptoms experience is excessive thirst, frequent urination, profuse sweating and in some case generalized itching.

High blood glucose levels can give to the formation of fatty deposits in the blood vessel walls; restricts the flow of blood and increases the risk for atherosclerosis that is the hardening of the blood vessels. People with diabetes are prone to foot problems due to the narrowing of blood vessels in the legs and feet. This problem can result in decreased sensation in the extremities and developed to a condition called diabetic neuropathy. Poor blood flow and nerve damage increase the chance of having a foot or leg amputated.

Monitoring: HBA1C levels show the mean blood glucose concentration over several weeks and months. Hence, monitoring of the mean blood glucose concentration can be tested every three months. It is an excellent method of determining compliance with medication, self-management and determining the risk for diabetes-related complications.

Medication and compliance: There are four main groups of insulin- fast acting, intermediate-acting, short-acting and long-acting. Insulin plays many roles in the body including the management of sugar levels in the blood. In the absence of insulin, the body is unable to use glucose as energy in the cells. This factor causes glucose to stay in the bloodstream and lead to high blood glucose or hyperglycemia. Insulin helps to control the 
blood glucose levels by sending the message to the liver, muscle and fat cells to take in glucose from the blood. On the other hand, if there is enough glucose the liver takes it and stores it as glycogen.

Additionally, besides glucose regulation, insulin plays other roles in the body as to change the activity of enzymes. Building of muscle following sickness or injury; this process is facilitated through the transportation of amino acids to muscle tissue, to repair muscular damage and to increase its size and strength. It also helps to regulate the uptake of amino acids, DNA replication, and synthesis of proteins.

Insulin manages the breakdown of protein and lipids due to changes in fat cells. It helps to uptake amino acids and potassium into cells that cannot take place in the absence of insulin. Insulin manages the excretion of sodium and fluid volume in the urine. Most importantly, it enhances learning and memory of brain functions.

Alpha-glycosidase inhibitors: The types of oral medications are recommended based on the health status. Alpha-glucosidase is one the enzymes responsible for the breaking down carbohydrates to smaller sugar particulars. The alpha-glucosidase inhibitors work by competitive and reversible inhibition of the intestinal enzymes. Hence, digestion of carbohydrates is slower and delay of glucose absorption. Therefore, there is smaller and slower rise in the blood glucose levels following meals, throughout the day.

Nutritional food groups: Macronutrients are essential nutrients that offer calories. Nutrients are substances needed for growth, metabolism and other functioning in large quantities. Categories of macronutrients are carbohydrates, proteins, and fats.

Our body also needed micronutrients and water. Micronutrients include vitamins and minerals. It is important for people with diabetes to understand what foods are made of, so they can better manage and control their blood glucose levels. It is important to eat foods from each of the food group daily.

Portions and servings: Too much food from any of the group will cause weight gain and high blood glucose levels. Serving sizes vary depending on the nutrition facts labels. Therefore, it is important to determine the caloric values and macronutrients based on the nutrition facts labels and recommended portions per servings from your Dietician.

Starch and starchy vegetable group: People with diabetes need to eat food from this group to help them to meet their daily requirement for calories, macronutrients, fibers, vitamins and minerals for good health. It is vital to space serving and to eat the required portions recommended. Starchy foods are healthy and have more calories. Hence, it is important to group them with vegetables. On the other hand, it is essential to

- Choose starches made with little fat regularly such as a slice of toast instead of a doughnut.

- Choose foods made from whole grains due to the fiber content such as whole wheat bread, grain pasta, and cereals.

- Always check the nutrition facts labels of foods to determine the servings from the starchy food group.

Tortillas vary in size, shape, color and texture; hence, it is imperative when counting calories and carbohydrates to self-manage your blood sugar levels. All tortillas are not of the same. It is vital when choosing them.

Fruit group: Fruits can be fresh, frozen, canned, dried and in the form of juices. Fruits are grouped together because the calories content come from carbohydrates and contain very little protein and no fat. Fruits are needed and are part of the meal plan.

Vegetable group: Non-starchy vegetables are rich in vitamins and minerals. Vegetables low in calories and fats are a good source of fiber. Vegetables are lower in carbohydrates. Larger servings can be eaten.

Fish, meat and substitutes group: The groups of food contain calories from protein and fat. Some meat substitutes and cheese contained small amounts of carbohydrate; the main macronutrients in these foods are protein and fat. Protein is essential to maintain muscles, 
Texila International Journal of Nursing

Volume 3, Issue 1, Mar 2017

make enzymes and keep the immune system working. Meat, eggs, and cheese are high in saturated fat and cholesterol.

Self-management of diabetes, people need to make heart-healthy choices when choosing food from this group due to the risk for increased cardiovascular complications.

Fats and oils group: Foods including butter, margarine, salad dressing, oils, and nuts just to name a few. These foods contain similar amounts of calories and fat per serving. Polyunsaturated, monounsaturated, trans and saturated fats are the chemical structure of different fats. Saturated and trans fats, studies showed the risk for increase heart disease.

Avoiding weight gain: Factors essential for managing weight are healthy eating habits and physical activities. Determining the body-mass index (BMI) is a tool used to measure the body fat based on height and weight. A BMI between 18.5 and 24.9 is considered normal, between 25 and 29.9 overweight and 30 and higher obese.

The body weight is regulated mainly by the number of calories consumed and burnt off. However, there are challenging factors such as stress, low-income levels, some medical conditions and medications that challenged weight gain. Too little or too much sleep, not enough physical exercise and too much alcohol consumption are contributed to weight gain.

- It helps to prevent or manage diabetes.

- It improves blood glucose levels, blood pressure, and blood lipids.

- It reduces the risk for complications such as heart disease and stroke

- It improves well-being and energy levels.

Sweeteners and sugar substitutes: Low calories sweeteners are called artificial sweeteners, sugar substitutes or non-nutritive sweeteners. They are used to sweeten food and drinks of lesser calories and carbohydrate to replaced sugar. Many foods labeled sugar-free, reduced sugar or no sugar added are not necessarily sugar-free; therefore, it is important to check nutrition facts. Sugar alcohols are known to produce side effects such as gas, bloating and abdominal discomfort. However, it is advisable to limit total added sugar intake and not to switch to sugar substitutes.

\section{The effects of alcohol}

- It stimulates the appetite and can cause overeating and interfere with the blood glucose level.

- Alcoholic drinks are high in calories and contribute to excessive weight gain.

- The effects of alcohol altered the sense of judgment causing poor food choices.

- Alcohol interferes with the effects of oral diabetes medication and insulin and causes hypoglycemic.

- Alcohol increases the levels of triglyceride.

- It also increases the blood pressure levels and causes flushing nausea and increased heart rate.

- Alcohol intake may also confuse or mask the symptoms of low blood sugar.

- Alcohol can cause blood glucose levels to rise or fall depending on the amount consumed and stimulate the pancreas to make more insulin.

- Combining the blood-sugar-lowering effects of the medication with alcohol intake can lead to hypoglycemia or insulin shock which is a medical emergency.

- Blood glucose levels are low up to about 12 hours of alcohol intake.

\section{Measures to be taken prior consuming alcohol}

- Be cautious and conservative if you are using insulin and test your blood sugar before consuming alcohol.

- Have a meal or snack with carbohydrates when you consume alcohol.

- Keep glucose or fast-acting glucose supplements with you at all times.

- Wear your medical identification band at all times (I am a Diabetic).

A step-wise approach to insulin dose adjustment: Type $2 \mathrm{DM}$ is characterized by progressive B-cell failure and escalating difficulties in maintaining glycemic control (Turner, 
2012). Scientific research has proven that possible cellular mechanisms are responsible for the decrease in B-cell function. Additionally, identification of allelic variants of several genes contributed to the risk of type $2 \mathrm{DM}$ development. The number of risk alleles increase and worsen many aspects of the B-cell function. The reaction to insulin reduces in a relative number of risk alleles ( $t$ 'Hart, 2010). The cellular mechanisms of risk alleles impair insulin secretion is not known. Despite the use of multiple oral anti-diabetes drugs, many people need insulin therapy to maintain normal levels of glycated hemoglobin (HbAlc).

The step-wise approach can be achieved through improved patient-provider communication; continuous discussion with clients and their relatives on the risk and benefits shared decision-making and educating clients in how to self-mange the disease and their insulin regime (Karter, 2010). Non-compliance resulted in the rise of the HbAlc levels which cause multiple drugs to be added to the medication regime and to improve glycemic control. Most of the times have the effect on clients such as loss of confidence, increased in emotional burden and the need for treatment compliance. Clients taking oral anti-diabetic drugs with high levels of $\mathrm{HbAlc}$ insulin therapy are the drug of choice.

Safety is essential when selecting the dose of mealtime insulin because at mealtime the insulin target is achieved. There are many barriers to initiation of insulin therapy. Many clients fear about injections and the risk of hypoglycemia, difficulties managing insulin therapy and the perceptions that insulin may impose life-restrictions. Some people felt that that insulin use indicates a greater severity of disease and failure of self-management.

Hyperglycemia: Hyperglycemia is high blood glucose or blood sugar level. It is likely to occur when the body produces too little insulin and or cannot use it effectively.

\section{Causes of hyperglycemia}

- Inadequate doses of insulin in cases of type $1 \mathrm{DM}$.

- Unhealthy eating habits.

- Inadequate exercise.

- Stressful situation.

\section{Signs and symptoms of hyperglycemia}

- High blood glucose level

- High level of sugar in the urine

- Increased thirst

- Frequent urination

- Dry mouth

The treatment and prevention of hyperglycemia: To avoid hyperglycemia quality selfmonitoring of blood glucose levels and compliance to drug therapy is essential. The management of high blood sugar is necessary to avert complications. The following plan is necessary to prevent hyperglycemia.

- Reassessment and restructuring of drug therapy are essential to prevent episodes of hyperglycemia. It is of great importance to consult with the medical team.

- A recommended dietary change that is eating a well-balanced meal is a vital part of taking care and managing diabetes. A meal plan will help in the process of selecting the correct food choice with the required caloric values.

- Regular physical activity is vital to managing diabetes.

- Regular blood glucose monitoring daily and treating high blood glucose helps to avoid problems associated with hyperglycemia. The blood glucose meter gives a direct measure of the glucose concentration at the time of the test to detect hyperglycemia or hypoglycemia. Training is necessary to prevent confusion and inappropriate actions. Urine testing is not as accurate as the blood glucose monitoring. Urine output depends on the client's renal threshold for glucose. It can be difficult for people who are visual impaired to read the urine strips. 
Texila International Journal of Nursing

Volume 3, Issue 1, Mar 2017

Hypoglycemia: Hypoglycemia is a condition characterized by abnormally low blood glucose or blood sugar levels lower than $70 \mathrm{mg} / \mathrm{dl}$. The condition is known as insulin reaction or insulin shock.

\section{Causes of hypoglycemia}

- Hypoglycemia occurs due to many reasons such as in diabetic persons who take too much insulin.

- Diabetic persons who took insulin but did not have their regular meal or did not eat on time.

- Diabetic persons who over exercise and insulin requirement at that time fell lower than usual.

- Medication-induced, alcohol abuse, liver diseases, and kidney disorders are other likely causes.

Signs and symptoms of hypoglycemia: The signs and symptoms are described as the "Whipple's Triad" a collection of three criteria that suggested an individual's is experiencing hypoglycemia.

- Signs and symptoms of hypoglycemia

- Low plasma glucose levels below $50 \mathrm{mg} / \mathrm{dl}$ at the time of onset

- Symptoms go away when glucose levels return to normal.

Treatment for hypoglycemia: There are two possible approaches for treatment

- Immediate treatment- that is treatment the abnormal low blood sugar attack.

- Treating the underlying cause.

Persons with a low blood sugar need to use sugar substances as soon as possible. Persons who are hungry need to eat to reduce the attack of hypoglycemia. It is important for diabetic persons to maintain their regular eating times. Stabilizing the blood glucose levels within 15 to 20 minutes is vital to prevent further complications.

Foot care: Foot care is essential for all diabetic clients. Foot self-examination, doctor check up, and many other things to do to keep your feet in good shape, improve circulation and identify any problems before it is severe. It is advisable to get your feet examine once per year and report any corns, calluses, sores, bruises, cuts, infections or foot pain. It is important to note that diabetic-related foot problems can worsen rapidly and are difficult to treat. Therefore, prompt attention is needed. Steps necessary for foot self-care

- Inspect your feet daily.

- Make sure you are aware of what should be done and should not be done to your feet.

- Visit you foot care specialist as soon as you recognize any problems with your feet.

Caring for your feet: Things to do to maintain healthy feet-

- Take care of your diabetes by working with your health care team to keep your blood glucose at a normal range.

- Check your feet every day for spots, cuts, swelling and blisters. A mirror can be used to check difficult areas or seek help.

- Be active and plan physical activities with your health team.

- Wear comfortable fitting shoes with stocks at all times.

- Wash your feet every day, dry them gently and check between the toes,

- Keep your feet moist and smooth.

- Trim toenails, when needed cut them straight across and file the edges.

- Protect feet from hot and cold and never use hot water bottles, heating pad or electric blanket which is likely to cause burns.

- Elevate feet when you are in the sitting position and do not cross them for long periods.

- Avoid smoking.

Compliance: Compliance with self-care activities is an essential component of managing diabetes. Successful outcomes depend on treatment adherence and the interconnectivity with health professionals. Adherence to therapy is defined as the extent to which the clients behave in taking their medication, following a diet plan, and executing lifestyle changes with agreed 
recommendations from healthcare providers. Medication, diet, exercise and lifestyle changes can only be achieved through adherence to the overall recommended regime. Compliance with self-care activities involved a set of behavioral actions to care on a daily basis. These actions involve engaging in positive lifestyle behaviors. Following a meal plan, daily physical activity, and taking of prescribed medication oral hypoglycemic or insulin, monitoring of blood glucose levels, responding to and self-treating diabetes related problems. Foot care guidelines, the quest for medical care for diabetes and or any other related health problems are critical activities.

The daily self-care regime aids in reducing the chances of developing long-term complications. Non-compliance to medication decreases glycemic control and leads to complications including microvascular and macrovascular diseases and altered lipid metabolism. Examples of microvascular disease are retinopathy, neuropathy and diabetic neuropathy (kidney disease).

Self-care management model: a small island perspective: St. Vincent and the Grenadines is a small island with natural vegetation. Mountainous in nature and its main crops are starchy foods such as eddoes, dasheen, plantain, sweet potatoes, bananas, and breadfruit. Hence, the daily meal plan contains high levels of carbohydrates. High intake of daily starchy foods is one of the factors for high incidences of diabetics. On the other hand, most of these people are farmers and of a low socio-economic status. Many people have little knowledge of diabetic and low intelligent quotients (IQ). This self-care management model was designed for people of low socio-economic status and underdeveloped countries.

Self-management relates to the tasks than an individual living with diabetes must undertake to live healthily. These tasks include gaining confidence to deal with the medical management, role management, and emotional management. To assume each task individuals must collaborate with health professionals, actively share in decision-making with the health team, monitors and manages signs and symptoms of his or her condition. These can be achieved through lifestyles modification that addresses risk factors and promotes health by focusing on prevention, early intervention and by assessing and supporting the confidence in the ability to use support services.

\section{Self-management support model}

Table 1. Showing the critical aspects of self-management

\begin{tabular}{|c|}
\hline Self-management support \\
\hline Decision-making support \\
\hline $\begin{array}{l}\text { Health information support } \\
\text { network }\end{array}$ \\
\hline Social support network \\
\hline Self-care Management Model \\
\hline
\end{tabular}

Self-management support: Self-care management is client centered. It is an ongoing process and provides the client with knowledge and skills necessary for self-care. The needs and goals are guided by evidence-based standards. The model aims to

- Establish a plan with the client and relatives ensuring that they receive full support.

- Design a self-management plan, the goals set to fit clients needs based on priorities, lifestyles and their conditions.

- Provide ongoing support to sustain a healthier lifestyle. 
Texila International Journal of Nursing

Volume 3, Issue 1, Mar 2017

- Set up support programs to ensure continued enhancement of self-management skills, behavioral strategies and metabolic improvement flexible for self-management interventions to the uniqueness of the person lifestyle.

- Encourage clients to attend as many educational sessions to improve their knowledge on managing the disease.

- Educate clients on the importance of autonomy and motivate them to start and value diabetes self-management behaviors.

- Educate client on the importance of compliance with all aspect of care and adherence to medication.

- Educate client on chronic illness care, choices, control, compliance, and consequences that are fundamental and clearly defined each aspect to the client.

- Ensure follow-up checks and evaluation of care.

Decision-making support: The decision-making process involves identifying and defining problems; to ascertain client's beliefs, thoughts, and feelings that may support or hinder their efforts. Health care professional set long-term goals that the clients work toward achieving. However, it is vital to allow the clients to choose and commit to making behavioral changes that will help in achieving long-term goals. On the other hand, these self-care behaviors must be adhered to ensuring effective decision-making and maintaining a healthy lifestyle.

- Healthy eating practice: choosing food from each of the food groups in the right proportion daily.

- Daily exercise or being active such as walking, playing games or gardening.

- Monitoring of blood glucose level daily and HbA1c level every three to four months. Other monitoring such as daily foot checks, observe for signs and symptoms of hyperglycemia and hypoglycemia and general monitor of health status.

- Compliance with care and of taking of prescribed medication.

- Problem-solving, seeks help and discuss any concerns with your loved ones or healthcare providers.

- Reducing risks and healthy coping are behavioral factors that must be attended to daily.

The client should be able to evaluate their efforts, identify what was learned, make the necessary changes in their behaviors, how to solve problems and to evaluate the process. It is imperative also, for the client to understand his/her roles as a decision-maker and on how to assume the responsibility for care.

Shared and improved decision-making involved the client, relatives and healthcare team; providing information to promote informed decision-making throughout the lifetime. Involving the client as an active partner with clinicians in the treatment process is an effective way in the decision-making support process. Health professionals should work together with their clients identifying, enacting decision and planning based on medical evidence. The decision-making process requires a civilization of collaborative work and skills that support the clients to think through and communicate their preferences. Also, to design and work with the necessary tools to make the decision-making process useful.

The aims of the decision-making support process are to

- Help clinicians and clients to work together as active partners to clarify acceptable medical options and to choose appropriate treatments.

- Acknowledge decision-making that may be influenced by knowledge, experience, personal situation, preferences, capabilities and values or beliefs.

- Assist the client in recognizing what was presented to clinicians and valuing the outcomes.

- Advocate for the client.

The collaborative effort improves health literacy and helps the client to read, understand, evaluate and use health information to make appropriate decisions about self-management. On the other hand, reduces the risk of hospitalizations, complications and make suitable lifestyle changes. 
Active involvement enhances knowledge, lessens anxiety and allows the client to feel in control of his/her care. Hence, evidence-based client's decision aids in facilitating the process of making informed decisions about self-management, complying with care and improves client's perception of the risk for complications.

Health support network: Health support networking is essential, collaborative and an integrated team approach to assist clients living with diabetes to assume an active role in their care. This aspect of the model allows the health professionals to implement various strategies and techniques, to provide adequate education on and to support the development of problemsolving skills in various aspects of diabetes management. In the planning process selfmanagement of diabetes education should be recognized and ongoing support the integral components of care. Therefore, self-management education is a continuous process of facilitating knowledge, skills and the necessary abilities for diabetes self-care. It is imperative for healthcare providers and other integrating teams to define their shared vision of diabetes care to enhance the process. The goals are

- Healthcare professionals should ably support clients while taking into account multiple physiological and personal factors.

- Develop collaborative daily self-management plan.

- Encourage, cajole and persuade clients to do their daily tasks.

- Inform clients on the daily choices made have a greater impact on their outcomes.

- The consequences and rights of decision-making accrue directly on clients.

- The rights and responsibly of clients managing their conditions in the way best suited to the context and culture of their lives.

- Listen attentively to clients, ask what their needs are and what they will like to receive.

- Ask clients how they are doing in reaching their self-selected goals and caring for their diabetes.

- Design or used the Health Information System (HIS) as a feedback method to inform healthcare teams involved in the treating and caring process of the client.

The HIS will inform healthcare providers on a complex set of behavioral actions, and daily activities implemented. The linkage will connect all healthcare providers such as the dietician, foot care practitioner, medical officer, dentist, laboratory, outpatient department, district hospital/clinic and other facilities that are necessary for the caring process. The goals of health support network are

- Coaching and partnering in the caring process.

- Offer referrals with helpful feedback.

- Close monitoring and follow-up care.

- Review and revise care plan as needed on clients' and healthcare providers' assessment and follow-up care recommended.

- Obtain ongoing information of therapeutic and behavioral options and clients concerns about each option.

- Collaborative approaches improve clients' outcomes.

- Creates a client-centered environment and receive support from all healthcare professionals.

Social support network: Social support is an intricate concept which coincides with multiple social networks such as the family, community, private and public sectors. Social support has the potential to exert positive influences on self-care management. However, the family plays a vital a role in the everyday instrumental tasks of diabetes care, especially in indigent and minority families. Social support is a process of interacting with others and comprised of emotional and informational support, positive and social interaction and has a significant impact on a person's physical and psychological wellbeing.

By helping people to cope with various situations, making them feel better about themselves by raising their self-esteem and improving their abilities and competently performing self-management task. Social support such as part of this self-care management model from a small island perspective can aid to improve health outcomes, cut cost due to 
Texila International Journal of Nursing

Volume 3, Issue 1, Mar 2017

limited and limited available resources. The main goal focuses on education which helps to support behavior change and improve self-management of people living with the long-term disease.

Family support, group interactions, community support groups, religious organization, health groups and counseling interventions are methods used to increase self-management. These interventions aid in improving self-monitoring, knowledge, and skills. Group involvement enhances learning and has effects on people with low IQ. There are many benefits achieved from social support networking such as

- Improved health behaviors such as exercise, consuming of nutritionally balanced diets and adherence to medical regimens. These factors promote health and prevent complications.

- Involvement with formal groups such religious organizations and informal groups such as friends and family these social ties help to improve the general wellbeing of clients. Social ties influence health behavior due to the influence or control, a spouse may have and their ongoing checks, reduce, regulate or facilitate healthy behaviors in many ways that will promote a partner's health and wellbeing. Social support network provides information and creates standards that influence and improves healthy habits.

Technology plays a significant role. Many devices are readily available and help to improve self-management. Technology can support daily diabetes self-management activities of blood glucose monitoring, exercising, and healthy eating, taking medication, monitoring for complication and problem-solving. The internet and smart phone can be used for educational and motivational support. Clinical information can be downloaded or recorded such as daily self-management activities. Visual activities improve clients' ability and problem-solving skills.

Technology has changed many lives, which can be motivational and allow for more frequent contact between healthcare providers and clients and enhance self-care management behaviors and treatment plans. Technology can be extended to clients' communities and homes and provide individualized care and just-in-time information.

\section{Diabetic take home card}

The diabetic take home card a guide designed to channel the way forward in selfmanagement, a holistic approach to self-care. The take home card guide is the ABC of managing diabetes. The card is the size of a passport. The content of the card is a reminder of what is to be done daily and records of clinical and medical visits, results of laboratory findings and documented care provided by the healthcare team.

\section{Simple take home card: a client guide}

Table 2. Representations of client take home card

\begin{tabular}{|c|c|}
\hline Page & Content \\
\hline Cover & $\begin{aligned} \text { I. } & \text { I am a diabetic } \\
\text { II. } & \text { Name of clinic } \\
\text { III. } & \text { Name of client } \\
\text { IV. } & \text { Contact and emergency numbers } \\
\text { V. } & \text { Present medication used }\end{aligned}$ \\
\hline
\end{tabular}




\begin{tabular}{|c|c|}
\hline Page 2 & $\begin{array}{l}\text { Monitoring blood glucose daily morning and evening and } \mathrm{HbA} 1 \mathrm{c} \text { level } \\
\text { quarterly }\end{array}$ \\
\hline Page 3 & $\begin{array}{l}\text { The best foods to be eaten } \\
\text { Vegetables: non-starchy: includes broccoli, carrots, greens, peppers, } \\
\text { and tomatoes. Starchy: includes potatoes, corn, and green peas. } \\
\text { Fruits: includes oranges, melon, berries, apples, bananas, and grapes } \\
\text { Grains: at least half of your grains for the day should be whole grains } \\
\text { such as wheat, rice, oats, cornmeal, barley, bread, pasta, and cereal. } \\
\text { Protein: lean meat, chicken or turkey without the skin, fish, eggs, nuts } \\
\text { and peanuts, dried beans, peas, such as chickpeas and split peas and } \\
\text { meat substitutes. } \\
\text { Dairy: nonfat or low fat, milk, yogurt, and cheese. Use oil instead of }\end{array}$ \\
\hline
\end{tabular}


Texila International Journal of Nursing

Volume 3, Issue 1, Mar 2017

\begin{tabular}{|l|l|}
\hline & butter. \\
\hline Page 4 & Physical activities \\
& $\begin{array}{l}\text { Daily foot care: wash dry and moist feet. Check between toes and } \\
\text { always wear your shoes. }\end{array}$ \\
\hline Page 5 & Daily medication plan- pill or injectable as prescribed. \\
\hline Page 6 & physical activities. \\
\hline Page 7 & Participation in educational activities/remarks \\
\hline Page 8 & Visits at district clinics/remarks \\
\hline Page 9 & Visits to practitioners/remarks \\
\hline Page 10 &
\end{tabular}

\section{Acknowledgement}

This publication was submitted as part of my requirement of my thesis; a partial fulfillment of the requirements for the award of Ph.D. in Nursing at the Texila American University (TAU). It is good to give thanks unto God for his goodness and loving kindness. With God all things are possible. On the other hand, special thanks to my immediate family for their encouragement, support, and prayers. Thanks to my colleagues for their support. Most importantly, I am thankful to Almighty God for bringing me thus far. Thank you, Lord, for guidance, protection, knowledge and the strength you continue to bestow unto me. Indeed he is a grateful God, the beginning, and the end.

\section{Conclusion}

Self-care has seen an evolutionary process of development or awareness living with the multifaceted nature of diabetes. Self-care management remains client centered and continues to be an ongoing process by providing the client with knowledge and skills necessary for selfcare. The needs and goals are guided by evidence-based standards. Family support, group interactions, community support groups, religious organization, health groups and counseling interventions continued to play pivotal roles in self-management. These interventions aided in improving self-monitoring, knowledge, and skills. Group involvement enhanced learning and has effects on people with low IQ.

There are many benefits achieved from social support networking especially immediate family support. Health support networking also remains essential, collaborative and an integrated team approach to help clients living with the disease to assume an active role in their care. This aspect of the model allowed health professionals to implement various strategies and techniques, provided adequate education and supports the development of problem-solving skills in various aspects of diabetes management. In the planning process, self-management education should be recognized and ongoing support the integral components of care.

Technology continues to play a significant role. Many devices are readily available and help to improve self-management. The decision-making process aided in identifying and defining problems; to find out client's beliefs, thoughts, and feelings that may support or hinder their efforts. Health care professional should identify long-term goals that are 
achievable. However, it is vital to allow the client to choose and commit to making behavioral changes that will help in achieving those goals. On the other hand, these self-care behaviors must be adhered to, ensure effective decision-making and maintaining a healthy lifestyle practices. The self-management clients take-home card, an excellent reminder of daily activities.

\section{References}

[1]. American Association Diabetes. (2009). Standards of Medial Care in Diabetes. Diabetes Care., Pp. 32.

[2]. American Association of Diabetes Educators. (2008). AADE7 Self-Care Behaviors. Diabetes Educ., Pp. 445-449.

[3]. American College of Endocrinology., (2002). The American association of clinical endicrinologist guidelines for the management of diabetes mellitus: the AACE system of diabetes self-management. Endocr Pract., Pp. S41-S84.

[4]. Attridge, M. C. (2014). Culturally appropriate health education for people in ethnic minority groups with type 2 diabetes mellitus. (3) Cochrane Database Syst. Rev., Pp. 9. CD006424. doi: 10 1002/14651858. CD006424. pub3.

[5]. Barlow, J. W. (2002). Self-management approaches for people with chronic illness: A review. Patient Education and Counseling., Pp. 177-187.

[6]. Cooper, H. B. (2003). Paitents' perspectives on diabetes health care education. Health Educ Res., Pp. 191-206.

[7]. Frost, J. G. (2014). A qualitative synthesis of diabetes self-management strategies for long term medical outcomes and quality of life in the UK. BMC Health Serv Res., Pp. 348. doi. 10. 1186/14726963-14-348.

[8]. Goodall, T. \&. (1991). Self-management of diabetes mellitus: a critical review. Health Psychol., Pp. 1-8.

[9]. Goodall, T. \&. (1991). Self-management of diabetes mellitus: a critical review. Health Psychol., Pp. 1-8.

[10]. Grady, J. E. (2011.). Using message framing to achieve long-term behavioral change in persons with diabetes. Apply Nurs Res., Pp. 22-28.

[11]. Hendra, J. \&. (1997). Improving the care of elderly diabetic patients: the final report of the St. Vincent joint task force. Age and Aging., Pp. 3-6.

[12]. Kater, A. S. (2010). Barriers to insulin initiation: the Translating Research Into Action for Diabetes insulin starts project. Diabetes Care., Pp. 33 (4): 733-735.

[13]. Matricciant, L. \&. (2015). Who cares about foot care? Barriers and enablers of foot self-care practices among non-institutionalized older adults diagnosed with diabetes: an integrative review. Diabetes Educ., Pp. 106-17.

[14]. Mc Nabb, W. (1997). Adherence in diabetes: can we define it and can we measure it? Diabetes Care., Pp. 215-218.

[15]. Mensing, C. B., \& cahy, K. \&. (2006). National standards for diabetes self-management education. Diabetes Care., Pp. 29 (Suppl 1): S78-S85.

[16]. Norris, S. L. (2002). Self-management education for adults with type-2 diabetes: a meta-analysis of the effect on glycemic control. Diabetes Care., Pp. 1159-1171.

[17]. Paterson, B. \&. (2000). Developmental evolution of expertise in diabetes self-management. Clin Nurs., Pp. 402-419.

[18]. Richard, A. \&. (2011). Delineation of self-care and associated concepts. Journal of Nursing Scholarship., Pp. 255-264.

[19]. Seuring, T. A. (2015). The economic costs of type 2 diabetes: a global systematic review. Pharmacoeconomics., Pp. 811-31.

[20]. Shobhana, R. B. (1999). Patients' adherence to diabetes treatment. Assoc Physicians India., Pp. 1173-1175. 
Texila International Journal of Nursing

Volume 3, Issue 1, Mar 2017

[21]. t'Hart, L. S.-B. (2010). Combined risk allele score of eight type 2 diabetes genes is associated with reduced first-phase glucose-stimulated insulin secretion during hyperglycaemic clamps. Diabetes., Pp. 59: 287-292.

[22]. Turner, R. C. (2005-2012). For the UK Prospective Diabetes Study (UKPDS) Group. Glycemic control with diet, sulfonylurea, metformin, or insulin in patients with type 2 diabetes mellitus: progressive requirement for multiple therapies (UKPDS 49). JAMA., Pp. 281 (21).

[23]. Williams, G. F. (1998). Supporting autonomy to motivate patients with diabetes for glucose control. Diabetes Care., Pp. 1644-1651.

[24]. World Health Organization. (1998). List of Basic Terms. Health Promotion Glossary., Pp. 4. 